\title{
Stress intensity factor measurements from digital image correlation: post-processing and integrated approaches
}

\author{
Stéphane Roux * \\ Surface du Verre et Interfaces \\ Unité Mixte de Recherche CNRS/Saint-Gobain \\ 39 quai Lucien Lefranc, F-93303 Aubervilliers Cedex, France.
}

(stephane.roux@saint-gobain.com)

François Hild

LMT-Cachan

ENS de Cachan / CNRS-UMR 8535 / Université Paris 6

61 avenue du Président Wilson, F-94235 Cachan Cedex, France.

(hild@lmt.ens-cachan.fr)

Abstract. Digital image correlation is an appealing technique for studying crack propagation in brittle materials such as ceramics. A case study is discussed where the crack geometry, and the crack opening displacement are evaluated from image correlation by following two different measurement and identification routes. The displacement uncertainty can reach the nanometer range even though optical pictures are dealt with. The stress intensity factor is estimated with a $7 \%$ uncertainty in a complex loading set-up without having to resort to a numerical modelling of the experiment.

Keywords: Brittle fracture, Ceramics, Photomechanics.
$\mathrm{SiC}$ - Silicon carbide;
SIF - Stress Intensity Factor;
SB - Sandwiched-beam

Abbreviations: COD - Crack opening displacement;

(C) 2005 Kluwer Academic Publishers. Printed in the Netherlands. 


\section{Introduction}

Fracture toughness is a key property for the design of fracture-safe structures, especially involving brittle materials [1]. With the development of full-field measurement techniques, the evaluation of the toughness may become similar to procedures used in numerical modeling such as finite element analyses [2-4]. Digital image correlation has already been utilized to analyze cracks in homogeneous and heterogeneous materials $[5,6]$. For instance, stress intensity factors $[7,8]$, crack tip opening angles [9] or crack tip opening displacements [10], and toughness [11] can be measured with a very good accuracy by means of digital image correlation. The application discussed herein is based upon a sandwiched-beam experiment $[12-14,11]$. This type of experimental set-up allows one to initiate a crack that does not propagate across the whole sample. However, the arrest conditions are strongly dependent upon the friction between the beams, their flexural rigidities, the notch geometry as well as the variations of material properties around the notch. Therefore, a toughness identification based upon finite element simulations was shown to be unreliable [11].

It is proposed to discuss the SIF identification based on displacements evaluated through digital image correlation by using two different ways:

- an a posteriori fit of displacements;

- an integrated approach in which the SIF is a direct output of the measurement stage.

\footnotetext{
* to whom correspondence should be addressed. Fax: +331 48347416 .
} 
It is worth noting that in both cases no finite element simulation is needed to evaluate the value of the stress intensity factor and the toughness. This fact allows the analysis to be applicable to a wide variety of cases.

Section 2 introduces the general correlation concepts used in the present study. The experimental procedure used to measure the toughness of brittle materials is based upon a sandwiched-beam technique (to precrack a notched beam) as discussed in Section 3. It is usually followed by a three-point flexural test on the precracked sample to determine the toughness of the tested specimen $[14,11]$. In the present case, the toughness is evaluated through the analysis of crack arrest for a silicon carbide ceramic using either a post-processing technique (Section 4) or an integrated procedure (Section 5) of the measured displacement field. The appendix presents an evaluation of the impact of image noise on the uncertainty of displacement evaluations for all procedures introduced in this study.

\section{Principle of image correlation}

The principle of image correlation techniques is the following. Two digital (gray level) images corresponding respectively to a reference, $f(\mathbf{x})$, and deformed, $g(\mathbf{x})$, state are simply related by the "passive" advection of the local texture in the displacement field $\mathbf{u}$. Therefore it is assumed that

$$
g(\mathbf{x})=f(\mathbf{x}+\mathbf{u}(\mathbf{x}))
$$


From the knowledge of $f$ and $g$, the problem consists in estimating $\mathbf{u}$ as accurately as possible, usually by regularization of the conservation equation of the optical flow [15-19].

\subsection{General approach}

Whenever $\mathbf{u}$ is a simple rigid translation, a powerful technique consists in looking for the maximum correlation between $f$ and $g$. The latter is reached accurately for the displacement $\mathbf{u}$, and suited interpolation techniques allows one to reach sub-pixel uncertainty (down to $10^{-2}$ pixel or below in suited cases [20]). From this observation a natural scheme is to decompose the original image into small zones of interest, where the approximation of a local rigid translation or uniform strain may hold. The collocation of all the local translations thus provides an estimate of the global displacement field. Each elementary determination can be performed either in the physical space $[21,22,20]$ or in Fourier space [23-25]. One difficulty however is the accurate pairing of zones of interest which implies a good evaluation of the displacement prior to further refinement. This has led to the development of multiscale algorithms that proceed by iteration from a coarse description to a finer one [26].

\subsection{EXtension to ARBITRARY FUnCTIONAL BASIS}

Recently a slightly different approach was suggested [27, 28]. Based on the same optical flow conservation principle, the minimization of $\|g(\mathbf{x})-f(\mathbf{x}+\mathbf{v}(\mathbf{x}))\|$ with respect to a trial displacement $\mathbf{v}(\mathbf{x})$, after a 
first order Taylor expansion leads to the minimization of

$$
\mathcal{T}[\mathbf{v}]=\iint[g(\mathbf{x})-f(\mathbf{x})-\mathbf{v}(\mathbf{x}) \cdot \nabla f]^{2} \mathrm{~d} \mathbf{x}
$$

A further expansion of the displacement onto a basis of chosen functions $\mathbf{v}(\mathbf{x})=\sum_{n} a_{n} \boldsymbol{\Psi}_{n}(\mathbf{x})$ allows one to write the amplitudes $a_{n}$ as the solution to the linear system

$$
M_{n m} a_{m}=b_{n}
$$

where

$$
\begin{gathered}
M_{n m}=\iint(\boldsymbol{\nabla} f \otimes \boldsymbol{\nabla} f):\left(\boldsymbol{\Psi}_{m} \otimes \mathbf{\Psi}_{n}\right) \mathrm{d} \mathbf{x} \\
b_{n}=\iint(f-g)\left(\boldsymbol{\nabla} f . \mathbf{\Psi}_{n}\right) \mathrm{d} \mathbf{x}
\end{gathered}
$$

The limitation of this approach comes from the applicability of the Taylor expansion (2). Hence, the maximum amplitude of the displacement that can be determined is typically less than one pixel. However, using a similar hierarchical approach as previously mentioned, based on progressive coarsening or filtering, allows one to progressively reach the required solution through gradual corrections of the deformed image with the determined estimate of the displacement field.

Let us note that the objective function provides a measure of the quality of the determination. Moreover, the integrand gives access to the local contribution of each pixel to the global error, and thus constitutes a very effective tool to appreciate the random or systematic nature of the residual error. 


\section{Application to a fracture test}

\subsection{SANDWICHED-BEAM EXPERIMENT}

To evaluate the fracture toughness of a brittle material (e.g., a silicon carbide ceramic), a sandwiched-beam precracking technique may be used $[12-14,11]$. The principle of an SB test consists in inserting a notched bar between two steel bars (see Fig. 1). The SB assembly is then loaded in three-point flexure. This type of experimental set-up allows one to initiate a crack that does not propagate across the whole sample. Consequently, the toughness evaluations are related to arrest conditions with the present technique. However, the arrest conditions are strongly dependent upon the friction between the beams, their flexural rigidities, the notch geometry as well as the variations of material properties around the notch. Furthermore, the load displacement curve has no precise signature corresponding to the onset of crack propagation. It was shown that digital image correlation could be used to analyze the experiment [11].

The material examined herein, called SiC-100 and made by Céramiques \& Composites (France), is naturally sintered. The powder is pressed and then heated to $2000{ }^{\circ} \mathrm{C}$. During processing, small quantities of boron carbide $\left(\mathrm{B}_{4} \mathrm{C}\right)$ are added to improve sintering. The average grain size is equal to $5 \mu \mathrm{m}$. Therefore, when artificial cracks are at least of millimetric size, a macroscopic evaluation of the stress intensity factor and toughness can be performed. The measurements carried out herein 
correspond to macroscopic evaluations of the SIF and toughness. The sample is notched with a diamond saw.

To achieve a good spatial resolution, a far-field (or long-distance) microscope is used to get access to the onset and arrest of crack propagation from a notch. The physical size $p$ of 1 pixel corresponds to $1.85 \mu \mathrm{m}$ so that a $1 / 100$-th pixel uncertainty is of the order of $20 \mathrm{~nm}$. A brightfield illumination is used in the present case and no special sample preparation is needed. At these magnification levels, the surface roughness provides enough variations so that "speckles" appear. Figure $2 \mathrm{~b}$ shows a picture taken when the load drop was observed. No crack is visible even with the highest magnification.

\subsection{Finite element Q1 functional basis}

Forquin et al. [11] studied the crack morphology and the mechanical characteristics using a standard correlation technique following the approach mentioned in Section 2.1. We thus defer to this reference for a detailed discussion of the methodology and results.

The same set of images was analyzed by following the generalized approach of Section 2.2, by using as a functional basis bilinear functions for each component of the displacement over square elements (Q1-shape functions in the language of the finite element method [29]). Even though the displacement uncertainty is the lowest for large element sizes, the inhomogeneity of the displacement prompts one to use small sizes. Sizes down to $6 \times 6$ pixels could be considered with a clear determination of the displacement. However, in order to reduce the 
uncertainty level (see next sub-section), elements of size $8 \times 8$ pixels were used in this section.

Figure 3 shows a map of the two components of the displacement field, where the discontinuity of displacement appears clearly, mainly in mode I, although the crack does not propagate along the vertical direction, had the experimental conditions been perfect. Consequently, an identification based on "perfect" boundary conditions, which is usually assumed by using finite element simulations, cannot be made.

\subsection{Performance}

The performance of the method in terms of standard displacement uncertainty is dependent on the texture of the image (e.g., gray level dynamic range, correlation length). To obtain an estimate of the uncertainty associated with the correlation algorithm, an artificial image is constructed from the true reference one (Fig. 2a) by using a sub-pixel translation in the range 0 to 1 pixel. (This is conveniently performed in Fourier space.) Then the correlation algorithm is applied to this pair of images, thus allowing for an evaluation of the uncertainty. A very rapid decrease of the uncertainty $\sigma_{u}$ can be observed with the size $\ell$ of the element (in pixels), as $\sigma_{u} \approx p A^{1+\alpha} \ell^{-\alpha}$ with $\alpha \approx 1.5$ and $A \approx 0.6$ pixel. (Note that $\ell$ is a pure number, and not a physical length scale). Thus for an element size of $\ell=8$ pixels as used below, $\sigma_{u} \approx 40 \mathrm{~nm}$, whereas for $\ell=128$ pixels, $\sigma_{u}$ is less than $1 \mathrm{~nm}$.

For the strain uncertainty, one can proceed along the same way and analyze artificial images where a homogeneous strain is imposed. This 
has been tested for a uniaxial strain ranging from $2.5 \times 10^{-4}$ to $8 \times 10^{-3}$. The most critical factor is the uncertainty (standard deviation around the estimated strain as a function of the imposed uniform strain) rather than the (much smaller) systematic error. For a small zone of interest $\ell=8$ pixels, the strain uncertainty amounts for about 8 times the value of the prescribed value (in the entire range). Requiring that the strain uncertainty be equal to the prescribed value would require $\ell \approx$ 20 pixels (more or less independently of the strain level). For a larger zone of interest, $\ell=128$ pixels, the relative uncertainty is reduced to about $12 \%$ over the entire strain range. It is worth mentioning that such uncertainties are significantly smaller than those obtained using classical digital image correlation techniques $[11,30]$.

Furthermore, the effect of noise due to the CCD camera on the displacement uncertainty is detailed in the appendix. For a given uncorrelated noise (characterized by a standard deviation $\sigma$ added to the picture $g$ ), it is shown that the standard displacement uncertainty $\tau_{u}$ (expressed in physical length units, rather than pixel size) associated to the analysis reads

$$
\tau_{u}=\frac{12 \sqrt{2} p \sigma}{7 \sqrt{\left\langle|\nabla f|^{2}\right\rangle} \ell}
$$

and therefore depends on properties of the analyzed texture. For the present application (Fig. 2a), one can compute $\sqrt{\left\langle|\nabla f|^{2}\right\rangle} \approx 17.2$ gray levels/pixel, and $\ell=8$ pixels, hence $\tau_{u} \approx 0.018 \sigma$. The temporal noise level $\sigma$ associated to image acquisition (i.e., the CCD camera) is difficult to estimate. The technical specification of the camera is such that the noise should be less than $3 \%$ of the 256 gray levels used, or about 8 gray levels. However, the residual error map that can be computed after 
the identification has to provide a true upper bound of the noise since it integrates all possible sources of discrepancies (including noise, but also all approximations due to interpolation, deviation from the ideal elastic field solution). As shown further down (see Fig. (8)), the latter residual (positive) error field has a mean level of 1.5 gray level. Thus in spite of the technical specification of the camera, we will use $\sigma=2$ gray levels here and in the sequel. The total displacement uncertainty $\tau_{u}$ is less than $75 \mathrm{~nm}$. It is worth noting that about $50 \%$ of that uncertainty is caused by the correlation technique itself as shown by the a priori performance estimate.

\section{Post processing analysis for toughness identification}

\subsection{Method}

Extracting some mechanically meaningful information using the detailed map of displacement can be performed by identifying the amplitudes of relevant reference displacement fields. In the present case, one can easily list some meaningful contributions. First, rigid body motions have to be accounted for, and constitute the first three fields, two inplane translations, $\boldsymbol{\Psi}_{1}$ and $\boldsymbol{\Psi}_{2}$, and one rotation about an axis normal to the observation plane, $\boldsymbol{\Psi}_{3}$. One single uniform strain field, $\boldsymbol{\Psi}_{4}$, is allowed for in order to leave the crack face stress-free, the so-called

"T-stress" (uniaxial tension along the crack direction). The presence of the crack generates two independent singular displacement fields corresponding to modes I, $\Psi_{5}$, and II, $\mathbf{\Psi}_{6}$, with two scalar amplitudes 
that will give access to the stress intensity factors. Last, in order to enrich the basis, one may also consider the next order sub-singular mode I and II fields, respectively denoted by $\boldsymbol{\Psi}_{7}$ and $\boldsymbol{\Psi}_{8}$. It is very convenient to express these vectors as complex valued fields in the local crack frame (crack tip at the origin, and crack extension along the negative real axis). A current point $M$ is represented by a complex number $z=x+i y$ or polar coordinates $z=r e^{i \theta}$, and similarly the displacement is represented by $\mathbf{U}=U_{x}+i U_{y}$. The expression of the eight basis functions is

$$
\begin{aligned}
& \mathbf{\Psi}_{1}(z)=1 \\
& \boldsymbol{\Psi}_{2}(z)=i \\
& \mathbf{\Psi}_{3}(z)=i z \\
& \mathbf{\Psi}_{4}(z)=(\kappa-1) z+2 \bar{z} \\
& \boldsymbol{\Psi}_{5}(z)=\sqrt{r}\left[2 \kappa e^{i \theta / 2}-e^{3 i \theta / 2}-e^{-i \theta / 2}\right] \\
& \mathbf{\Psi}_{6}(z)=i \sqrt{r}\left[2 \kappa e^{i \theta / 2}+e^{3 i \theta / 2}-3 e^{-i \theta / 2}\right] \\
& \boldsymbol{\Psi}_{7}(z)=\sqrt{r^{3}}\left[2 \kappa e^{3 i \theta / 2}-3 e^{i \theta / 2}+e^{-3 i \theta / 2}\right] \\
& \mathbf{\Psi}_{8}(z)=i \sqrt{r^{3}}\left[2 \kappa e^{3 i \theta / 2}+3 e^{i \theta / 2}-5 e^{-3 i \theta / 2}\right]
\end{aligned}
$$

where

$$
\kappa=\frac{(3-\nu)}{(1+\nu)}
$$

in plane stress condition, as expected along the free observation surface, with $\nu$ being the Poisson's ratio. Let us note however that the amplitudes of these functions are real numbers.

The strategy is thus simply to decompose the estimated displacement field (from image correlation) onto the basis of $\boldsymbol{\Psi}_{k}$ test functions. 
For this goal, the following objective function is minimized

$$
\mathcal{T}(\mathbf{a})=\sum_{i=1}^{N_{\text {mes }}}\left\|\mathbf{U}_{i}-\sum_{k} a_{k} \mathbf{\Psi}_{k}\right\|^{2}
$$

where $N_{\text {mes }}$ is the number of measured displacement points.

One advantage of this formulation is that the value reached by the objective function constitutes a global quality parameter. Since the identification procedure assumes that the crack geometry is known, a minimization of this global residual over the a priori guessed crack tip position also provides a natural way of optimizing the crack geometry. In the following, in order to make this estimator dimensionless, we will refer to $\left(\mathcal{T}-\mathcal{T}_{\text {min }}\right) / \mathcal{T}_{\text {min }}$ as the "global residual".

It can be noted that other techniques can be followed to extract the stress intensity factor by computing the crack opening displacement profile [11] or by using an interaction integral [8].

\subsection{Results}

The results reported below are obtained from the previous estimates of the displacement field resolved with a mesh size of 8 pixels, over a region of interest that amounts to $512 \times 512$ pixels centered on a gross determination of the crack tip position. In Fig. 4, the map of estimated mode I and mode II stress intensity factors is plotted as functions of the guessed crack tip location. To select the most appropriate location, the map of global residual is shown in the same figure. The crack tip can be accurately located in the direction normal to its mean orientation, whereas along its extension the residuals vary slowly. Yet, from the absolute minimum location, one can locate quite precisely the 
crack tip position that provides the best fit quality. The uncertainty is about \pm 10 and \pm 2 pixels respectively parallel or perpendicular to the crack direction (i.e., about 20 and $4 \mu \mathrm{m}$ ). This uncertainty is based on the subjective appreciation of extreme crack tip positions that can be considered as unacceptable.

More importantly, the same analysis also provides a quantitative estimate of the stress intensity factors for both modes I and II. The singular field, which is directly measured through the amplitudes of $\Psi_{5}$ and $\Psi_{6}$, yields the SIF when the elastic constants are known (i.e., for $\mathrm{SiC}, E=410 \mathrm{GPa}$ and $\nu=0.15$ ). One obtains $K_{I}=2.3 \pm 0.2$ $\mathrm{MPa} \sqrt{\mathrm{m}}$, and $K_{I I}=0.0 \pm 0.05 \mathrm{MPa} \sqrt{\mathrm{m}}$. Error bars are estimated from the values of $K$ for extreme locations of the crack tip location as indicated above.

The derivation presented in the appendix provides the value of the identification uncertainties associated to the noise of the CCD camera $\sigma$ as

$$
\begin{aligned}
& \tau(\text { translation }) \approx 0.42 \sigma(\mathrm{nm}) \\
& \tau(\text { rotation }) \approx 1.7 \times 10^{-6} \sigma(\mathrm{rad}) \\
& \tau(\mathrm{SIF}) \approx 0.01 \sigma(\mathrm{MPa} \sqrt{\mathrm{m}})
\end{aligned}
$$

when $\sigma$ is expressed in gray levels. The previous estimate for $\sigma$ (i.e., less than 2 gray levels) gives the identification uncertainty for the stress intensity factor as equal to $0.02 \mathrm{MPa} \sqrt{\mathrm{m}}$.

This estimate is computed provided the geometry of the medium (including crack tip location) is known. However the dominant source of uncertainty comes from this unknown geometry, and using a somewhat 
subjective evaluation, error bars on $K$ are estimated as 10 times as large (as values mentioned above) in mode I, and 2.5 times in mode II.

Despite the fact that the crack started at an angle with respect to the expected vertical direction, the singular crack displacement is essentially under a mode I condition, with very little tangential displacement discontinuity. In quantitative terms, the mode I opening is about 0.3 pixel wide (i.e., about $500 \mathrm{~nm}$ ) in the region of interest, whereas for mode II, the tangential discontinuity is at most of order 0.006 pixel, or about $10 \mathrm{~nm}$ at the edge of the region of interest.

\section{Integrated approach}

\subsection{Methodology}

Up to now, the identification procedure consisted of two independent steps:

- first, the identification of the displacement field (Section 2), and in particular the use of an arbitrary functional basis for that purpose,

- second, the projection of the measured displacement field onto a few mechanically significant fields (Section 4).

It can be noticed that this separation into two steps is unnecessary, and in particular the introduction of Q1-elements is artificial, and only used at an intermediate stage. This a priori partition will reduce the performance of the method, as compared to what is now introduced 
and referred to as an "integrated approach." The route outlined in Subsection 2.2 is still followed, but the relevant displacement fields, $\boldsymbol{\Psi}_{i}$, are introduced a priori at the measurement stage. The direct computation of the amplitudes of the singular crack fields will thus immediately provide an estimate for the SIFs. The generality of the correlation approach is fully exploited, and no specific restrictions appear from having to deal with the eight functions that were used before [Eqn. (7)]. The same multiscale strategy holds since it only involves a coarse-graining or filtering of the images and does not imply specific limitations on the displacement fields. Sub-pixel corrections may be introduced to iteratively reach the optimal determination, although we did not use any correction on anything but the mean displacement (of about 11 pixels in magnitude along the loading direction).

\subsection{Results}

The integrated approach is applied to study the same experiment, and the same pair of pictures. A zone of interest of size $512 \times 512$ pixels, approximately centered on (an estimate of) the crack tip, is used in the analysis. Here again, the crack tip is estimated through the minimum value of an objective function.

Figure 6 shows the optimum stress intensity factors for modes I and II, as functions of the assumed crack tip position, and the global residual (relative to its minimum value) is also shown in the same figure. A very close similarity is observed between the latter results and the ones obtained using the post-processing step on the estimated 
displacement. The same conclusion holds for the best determination of the displacement fields shown in Fig. 7. In more quantitative terms, the critical stress intensity factors are estimated to amount to

$$
\begin{aligned}
& K_{I}=2.3 \pm 0.15 \mathrm{MPa} \sqrt{\mathrm{m}} \\
& K_{I I}=0.05 \pm 0.05 \mathrm{MPa} \sqrt{\mathrm{m}}
\end{aligned}
$$

Again, error bars are subjectively estimated by extracting extreme positions of crack tip that would be acceptable, and measuring the corresponding change in $K$.

As mentioned earlier, the objective function $\mathcal{T}$ provides naturally a map of local contributions to residual errors. Such an error field after the integrated approach is shown in Fig. 8. The units are in gray levels. Even though the error level can be locally as large as 30 gray levels (for an 8-bit digitization, or 256 gray levels, of the initial images), the mean value of the residual error is about 1.5 gray levels. This value was used in order to obtain an upper bound for the noise level $\sigma=2$ gray levels. The error is highly concentrated along the crack path as could have been anticipated. One also sees coherent zones of high error values (e.g., in the right hand top corner of this figure), which corresponds to deviations from the crack displacement field, presumably due to a subsuperficial porosity.

Let us note that these estimates of the SIF are in excellent agreement with the previous determination using the post-processing treatment based on the Q1-based estimates of the displacement field. The very close similarity of the quality factor and SIF values as functions of the assumed position of the crack tip (Figs. 4 and 6) is striking. Consequently the estimated displacement fields using both post-processing 
(Fig. 5) and integrated approaches (Fig. 7) are extremely close in terms of mean value.

The uncertainty with the integrated approach is quite comparable to that observed with the post-processing analysis. However, that may not be a general statement. Because the projection over the Q1 finite element basis is an unnecessary step, the integrated approach will always have better or equal performances to those of post-processing routes. In the present case, because of the low level of noise, and the wide analyzed field, such a projection does not involve a drastic loss of information. The uncertainty associated to the CCD camera can again be evaluated by following the derivations in the appendix. The identification uncertainties are obtained as

$$
\begin{aligned}
& \tau(\text { translation }) \approx 0.4 \sigma(\mathrm{nm}) \\
& \tau(\text { rotation }) \approx 1.5 \times 10^{-6} \sigma(\mathrm{rad}) \\
& \tau(\mathrm{K}) \approx 0.009 \sigma(\mathrm{MPa} \sqrt{\mathrm{m}})
\end{aligned}
$$

when $\sigma$ is expressed in gray levels. These values are very close to those achieved by the post-processing approach (Section 4). Uncertainties for the displacement is of the order of $1 \mathrm{~nm}$, and for a SIF $\tau(K) \approx 2 \times 10^{-2} \mathrm{MPa} \sqrt{\mathrm{m}}$. Once again, unfortunately, the dominant error comes from the unknown geometry of the crack, which motivates the much more conservative error bars [see Eqn. (11)]. 


\section{Conclusion}

Theoretical and operational correlation tools that allow one to extract meaningful mechanical information from pictures have been presented. Crack openings much smaller than the pixel size, and even smaller than the wavelength of light, can be resolved thereby enabling for the determination of the crack tip with a small uncertainty, and even the identification or the measurement of stress intensity factors. In addition, the appendix provides a general methodology for estimating the impact of image noise on the uncertainty in the determination of displacements as well as stress intensity factors.

These results have been achieved through significant improvements over the standard performance of digital image correlation, based on projection of the displacement field over Q1-shape functions. The postprocessing step allows one to filter out fluctuations in the estimates of the displacement, and to obtain measurements of the stress intensity factors in the present example. Let us underline that this scheme, based upon the use of the Kolossov and Muskhelichvili potentials, is much more general and can easily be applied to a wide class of situations, naturally giving rise to a mechanically admissible displacement field.

An integrated approach, which avoids the artificial step of the projection onto a Q1-basis, is proposed. Even if the latter method is expected to be more accurate than the first two-step procedure, the obtained estimates are quite close, thereby cross-validating both approaches. This integrated technique can be extended to a large class of homogeneous elastic solids subjected to complex loadings. 


\section{Acknowledgements}

The experiments reported herein were carried out by Drs. P. Forquin and Y. Charles. This work was performed within the research network "GDR 2519 : Mesure de champs et identification en mécanique des solides."

\section{References}

1. B. R. Lawn, Fracture of Brittle Solids, (Cambridge University Press, Cambridge (UK), 1993).

2. Barsoum, R.S.: 1976, 'On the Use of Isoparametric Finite Elements in Linear Fracture Mechanics,' Int. J. Num. Meth. Eng. Vol. 10, pp. 25-37.

3. Barsoum, R.S.: 1977, 'Triangular Quarter-Point Elements as Elastic and Perfectly-Plastic Crack Tip Elements,' Int. J. Num. Meth. Eng. Vol. 11, pp. $85-98$.

4. Lim, I.L., and I.W. Johnston: 1992, 'Comparison Between Various Displacement-Based Stress Intensity Factor Computation Techniques,' Int. J. Fract. Vol. 58, pp. 193-210.

5. Rastogi, P.K.: Photomechanics, Springer, Berlin (Germany), 2000.

6. Anbanto-Bueno, J., and J. Lambros: 2002, 'Investigation of crack growth in functionally graded materials using digital image correlation,' Eng. Fract. Mech. Vol. 69, pp. 1695-1711.

7. McNeill, S.R., W.H. Peters, and M.A. Sutton: 1987, 'Estimation of stress intensity factor by digital image correlation,' Eng. Fract. Mech. Vol. 28 no. 1, pp. 101-112. 
8. Réthoré, J., A. Gravouil, F. Morestin, and A. Combescure: 2005, 'Estimation of mixed-mode stress intensity factors using digital image correlation and an interaction integral,' Int. J. Fract. Vol. 132, pp. 65-79.

9. Dawicke, D.S., and M.S. Sutton: 1994, 'CTOA and Crack-Tunneling Measurements in Thin Sheet 2024-T3 Aluminum Alloy,' Exp. Mech. Vol. 34, pp. 357-368.

10. Sutton, M.A., S.R. McNeill, J.D. Helm, and Y.J. Chao, Advances in TwoDimensional and Three-Dimensional Computer Vision, in: P.K. Rastogi, eds., Photomechanics, Springer, Berlin (Germany), 2000, pp. 323-372.

11. Forquin, P., L. Rota, Y. Charles, and F. Hild: 2004, 'A Method to Determine the Toughness Scatter of Brittle Materials,' Int. J. Fract. Vol. 125 no. 1, pp. 171-187.

12. Nose, T., and T. Fujii: 1988, 'Evaluation of Fracture Toughness for Ceramic Materials by a Single-Edge-Precracked-Beam Method,' J. Am. Ceram. Soc. Vol. 71 no. 5, pp. 328-333.

13. Marshall, D.B., J.J. Ratto, and F.F. Lange: 1991, 'Enhanced Fracture Toughness in Layered Microcomposites of Ce- $\mathrm{ZrO}_{2}$ and $\mathrm{Al}_{2} \mathrm{O}_{3}$, ' J. Am. Ceram. Soc.

Vol. 74 no. 12, pp. 2979-2987.

14. Pancheri, P., P. Bosetti, R. Dal Maschio, and V.M. Sglavo: 1998, 'Production of Sharp Cracks in Ceramic Materials by Three-Point Bending of Sandwiched Specimens,' Eng. Fract. Mech. Vol. 59 no. 4, pp. 447-456.

15. Horn, B.K.P., and B.G. Schunck: 1981, 'Determining optical flow,' Artificial Intelligence Vol. 17, pp. 185-203.

16. Mitiche, A., and P. Bouthemy: 1996, 'Computation and analysis of image motion: A synopsis of current problems and methods,' Int. J. Comp. Vision. Vol. 19, pp. 29-55.

17. Black, M.: Robust Incremental Optical Flow, PhD dissertation, Yale University, 1992.

18. Odobez, J.-M., and P. Bouthemy: 1995, 'Robust multiresolution estimation of parametric motion models,' J. Visual Comm. Image Repres. Vol. 6, pp. 348-365. 
19. Bogen, D., and D. Rahdert: 1996, 'A strain energy approach to regularization in displacement field fits of elastically deforming bodies,' IEEE Trans. Pattern Analysis and Machine Intelligence Vol. 18, pp. 629-635.

20. Sutton, M.A., S.R. McNeill, J. Jang, and M. Babai: 1988, 'Effects of subpixel image restoration on digital correlation error estimates,' Opt. Eng. Vol. 27 no. 10, pp. $870-877$.

21. Sutton, M.A., W.J. Wolters, W.H. Peters, W.F. Ranson, and S.R. McNeill: 1983, 'Determination of Displacements Using an Improved Digital Correlation Method,' Im. Vis. Comp. Vol. 1 no. 3, pp. 133-139.

22. Chu, T.C., W.F. Ranson, M.A. Sutton, and W.H. Petters: 1985, 'Applications of Digital-Image-Correlation Techniques to Experimental Mechanics,' Exp. Mech. Vol. 3 no. 25, pp. 232-244.

23. Chen, D.J., F.P. Chiang, Y.S. Tan, and H.S. Don: 1993, 'Digital SpeckleDisplacement Measurement Using a Complex Spectrum Method,' Appl. Opt. Vol. 32, pp. 1839-1849.

24. Berthaud, Y., J. Scholz, and J. Thesing, Méthodes optiques et acoustiques de mesures des caractéristiques mécaniques, Proc. Colloque national MECAMAT "Mécanismes et mécanique des grandes déformations," 1996, pp. 77-80.

25. Chiang, F.P., Q. Wang, and F. Lehman, New Developments in Full-Field Strain Measurements Using Speckles, in: Non-Traditional Methods of Sensing Stress, Strain and Damage in Materials and Structures, ASTM, Philadelphia (USA), 1997, pp. 156-169.

26. Hild, F., B. Raka, M. Baudequin, S. Roux, and F. Cantelaube: 2002, 'MultiScale Displacement Field Measurements of Compressed Mineral Wool Samples by Digital Image Correlation,' Appl. Optics Vol. IP 41 no. 32, pp. 6815-6828.

27. Roux, S., F. Hild, and Y. Berthaud: 2002, 'Correlation Image Velocimetry: A Spectral Approach,' Appl. Optics Vol. 41 no. 1, pp. 108-115.

28. Wagne, B., S. Roux, and F. Hild: 2002, 'Spectral Approach to Displacement Evaluation From Image Analysis,' Eur. Phys. J. AP Vol. 17, pp. 247-252.

29. Zienkievicz, O.C., and R.L. Taylor, The Finite Element Method, McGraw-Hill, London (UK), 4th edition, 1989. 
30. Bergonnier, S., F. Hild, and S. Roux: 2005, 'Strain heterogeneities in tension and compression tests on mineral wool samples,' J. Strain Analysis Vol. 40, pp. 185-197.

31. Holst, G., CCD Arrays, Cameras and Displays, SPIE Engineering Press, Washington DC (USA), 1998. 


\section{Appendix: Sensitivity to image noise}

Let us investigate the sensitivity of the result to noises associated to the image acquisition (e.g., digitization, read-out noise, black current noise, photon noise [31]). It is assumed that the deformed image is polluted by a random white noise $\eta$, of zero mean, and variance $\sigma^{2}$. (In practice, both reference and deformed images are affected by the same noise, however, this is equivalent to considering a noise in the difference $(f-g)$ of variance $2 \sigma^{2}$.) The notations of Section 2 are used. The $\mathbf{M}$ matrix is thus unaffected by this noise, but only the vector $\mathbf{b}$ is modified by a quantity

$$
\delta b_{n}=\iint \eta(\mathbf{x}) \cdot\left(\boldsymbol{\nabla} f \cdot \mathbf{\Psi}_{n}\right) \mathrm{d} \mathbf{x}
$$

On average, $\langle\delta \mathbf{b}\rangle=0$, and

$$
\left\langle\delta b_{m} \delta b_{n}\right\rangle=\sigma^{2} M_{m n}
$$

The impact of this noise on the determination of $\mathbf{a}$, which is not altered by a fluctuating part $\delta \mathbf{a}$, is sought. By linearity, one observes that $\langle\delta \mathbf{a}\rangle=0$. Its variance is given by

$$
\left\langle\delta a_{m} \delta a_{n}\right\rangle=\sigma^{2} M_{m p}^{-1} M_{n q}^{-1} M_{p q}=\sigma^{2} M_{m n}^{-1}
$$

The above analysis can be applied to all of the procedures, be it the Q1 estimate of the displacement field, the projection of this field on the 8-dimensional functions (where the notations are to be revisited but the algebra remains the same), or the integrated approach.

Let us start of with the Q1 estimate of the displacement field. Rather than computing the exact above estimate of the variance of 
the displacement field (the $\mathbf{M}^{-1}$ matrix is quite large), one can further simplify the computation, and only consider the spatial average of the covariance matrix $\left\langle\delta a_{m} \delta a_{n}\right\rangle$. This hypothesis ignores the edge effects where the variance will be larger than within the domain. It also exploits the assumption that the gradient of the image has only short distance correlations as compared to the Q1-element size. The average symbol $\langle\ldots\rangle$ is now understood as representing an ensemble average over the noise, and a spatial average. As a result the matrix $\mathbf{M}$ has to be averaged, and reads

$$
\left\langle M_{m n}\right\rangle=(1 / 2)\left\langle|\nabla f|^{2}\right\rangle \iint \boldsymbol{\Psi}_{m} \cdot \boldsymbol{\Psi}_{n} \mathrm{~d} \mathbf{x}
$$

The interesting feature of this equation is the fact that only the shape functions are involved in the integral. This results in a natural decoupling between the $x$ and the $y$ coordinates of the displacement, and a quasi-band diagonal of this function inherited from the classical finiteelement formulation. For instance, the diagonal elements will have the following expression

$$
\left\langle M_{n n}\right\rangle=(2 / 9)\left\langle|\nabla f|^{2}\right\rangle \ell^{2}
$$

for a summation performed over the 4 elements attached to this node. For two adjacent nodes, $\left\langle M_{m n}\right\rangle=(1 / 9)\left\langle|\nabla f|^{2}\right\rangle \ell^{2}$, and for two diagonally opposed nodes, $\left\langle M_{n n}\right\rangle=(1 / 36)\left\langle|\nabla f|^{2}\right\rangle \ell^{2}$. This matrix can easily be inverted. It has the same support as $\mathbf{M}$. The diagonal element is

$$
\langle M\rangle_{n n}^{-1}=\frac{288}{49\left\langle|\nabla f|^{2}\right\rangle \ell^{2}}
$$

The adjacent node matrix element is $-(1 / 4)$ that value, and diagonally opposed nodes are 1/16 of it. By making a final additional hypothesis 
that the inverse of the spatial average can be identified with the spatial average of the inverse (i.e., valid for a homogeneous texture of the image), the final expression of the standard deviation $\tau_{a}$ of the Q1 displacement amplitude is expressed as

$$
\tau_{a}=\sqrt{\left\langle\delta a_{n}^{2}\right\rangle}=\frac{12 \sqrt{2} p \sigma}{7 \sqrt{\left\langle|\nabla f|^{2}\right\rangle} \ell}
$$

where the physical size of a pixel, $p=1.85 \mu \mathrm{m}$, has been introduced to express $\tau_{a}$ in length units rather than pixel size. For the present application, one can compute $\sqrt{\left\langle|\nabla f|^{2}\right\rangle} \approx 17.2$ gray levels/pixel, and $\ell=8$ pixels, hence $\tau_{a} \approx 0.018 p \sigma$. It is to be noted that the fluctuating part of the a field is anti-correlated. Thus part of the fluctuations at one node can be compensated by the fluctuations on neighboring nodes. If a projection is to be performed on a field that is slowly varying over the distance $\ell$ then the "trace" (sum over all nodes) of $\mathbf{M}^{-1}$ is to be considered, so that this trace gives rise to a standard deviation of a that is only half of the above estimate, i.e., $0.009 \sigma$. To validate the various approximations performed above, one resorted to a sampling evaluation of the previous terms (1000 random fields were used). The results lead to $\tau_{a} \approx 0.014 p \sigma$ and for the trace $0.009 p \sigma$, in good agreement with the a priori estimates.

Let us now apply this analysis to the post-processing of the Q1 analysis. In this case, we revert to Section 4 , where the 8 amplitudes of the displacement fields are computed from the result of the Q1displacement. The noise $\eta$ and the $\mathbf{M}$ matrix introduced in the present section are now respectively the fluctuation $\delta \mathbf{a}$ of the previous discussion, and the sum over the domain of $\boldsymbol{\Psi}_{m}\left(\mathbf{x}_{i}\right) \boldsymbol{\Psi}_{n}\left(\mathrm{x}_{i}\right)$. Because one has a 
simple $8 \times 8$ matrix, a direct inversion can easily be performed and thus one obtains the complete covariance matrix of the different amplitudes. Symmetries are such that no off-diagonal term is significantly different from 0 . By considering only diagonal terms for $\mathbf{M}^{-1}$ and the previous direct estimate $\tau_{a} \approx 0.014 p \sigma$, the following standard deviations are expressed with their physical dimension (using $p=1.85 \mu \mathrm{m}$ ) and $\sigma$ is estimated in gray levels

$$
\begin{aligned}
& \tau(\text { translation }) \approx 0.42 \sigma(\mathrm{nm}) \\
& \tau(\text { rotation }) \approx 1.7 \times 10^{-6} \sigma(\mathrm{rad}) \\
& \tau(\mathrm{SIF}) \approx 0.01 \sigma(\mathrm{MPa} \sqrt{\mathrm{m}})
\end{aligned}
$$

Last, let us apply the above analysis to the integrated approach (Section 5). Here again, the final matrix of the linear system is $8 \times 8$, and thus one resorts to a direct evaluation of $\mathbf{M}^{-1}$ to obtain

$$
\begin{aligned}
& \tau(\text { translation }) \approx 0.4 \sigma(\mathrm{nm}) \\
& \tau(\text { rotation }) \approx 1.5 \times 10^{-6} \sigma(\mathrm{rad}) \\
& \tau(\mathrm{SIF}) \approx 0.009 \sigma(\mathrm{MPa} \sqrt{\mathrm{m}})
\end{aligned}
$$

It is interesting to note that the above estimates for the uncertainties of both procedures used to evaluate the stress intensity factors give very close estimates. Let us however underline that those uncertainties are based on an ideal noise and do not consider systematic bias due for instance to the projection of ideal crack displacement fields on a Q1-basis.

The temporal noise level of a given CCD camera depends upon a number of external parameters. The technical specification of the camera indicates that it should be in the present case below $3 \%$ of the 256 gray-levels used in the images, or about 8 gray levels. When using 
the residual error obtained after an analysis, (see Fig. 8) one observes that its mean value is about 1.5 gray levels. Since the latter is an upper bound that includes all possible sources of discrepancies (among which temporal noise is just one part), we consider that it provides a more reliable upper bound estimate of the noise, and thus $\sigma=2$ gray levels is selected. This indicates that the standard uncertainty resulting from the noise amounts to about $1 \mathrm{~nm}$ for a mean translation, and 0.02 $\mathrm{MPa} \sqrt{\mathrm{m}}$ for the SIF of the studied material. 


\section{List of Figures}

1 Schematic view of the SB bend test. The observation face of the $\mathrm{SiC}$ sample is shown in gray.

2 Eight-bit pictures of silicon carbide sample surface before (a) and after (b) loading. The notch on the lower edge of the sample is clearly visible ( 1 pixel $\leftrightarrow 1.85 \mu \mathrm{m}) . \quad 31$

3 Horizontal (a) and vertical (b) components of the displacement field expressed in pixels and resolved using Q1 elements of size $8 \times 8$ pixels (1 pixel $\leftrightarrow 1.85 \mu \mathrm{m}$ ).

$4 \quad K_{I}$ in $\mathrm{MPa} \sqrt{\mathrm{m}}(\mathrm{a}), K_{I I}$ in $\mathrm{MPa} \sqrt{\mathrm{m}}$ (b) and global residual $\left(\mathcal{T}-\mathcal{T}_{\min }\right) / \mathcal{T}_{\min }(\mathrm{c})$ as functions of the assumed crack tip position $x$ and $y$. The optimal position is depicted by the white cross.

5 Horizontal (a) and vertical (b) components of the displacement field expressed in pixels as projected onto the basis of suited functions in a post-processing of the measured displacements ( 1 pixel $\leftrightarrow 1.85 \mu \mathrm{m})$.

$6 \quad K_{I}$ in $\mathrm{MPa} \sqrt{\mathrm{m}}(\mathrm{a}), K_{I I} \mathrm{MPa} \sqrt{\mathrm{m}}(\mathrm{b})$ and global residual $\left(\mathcal{T}-\mathcal{T}_{\min }\right) / \mathcal{T}_{\min }(\mathrm{c})$ as functions of the assumed crack tip position $x$ and $y$.

7 Horizontal (a) and vertical (b) components of the displacement field expressed in pixels and identified by using the integrated approach $(1$ pixel $\leftrightarrow 1.85 \mu \mathrm{m})$. 
8 Residual error map for the integrated approach. The spatial integral of the square of this field gives the objective function to be minimized. The mean value of local error is about 1.5 gray levels. 


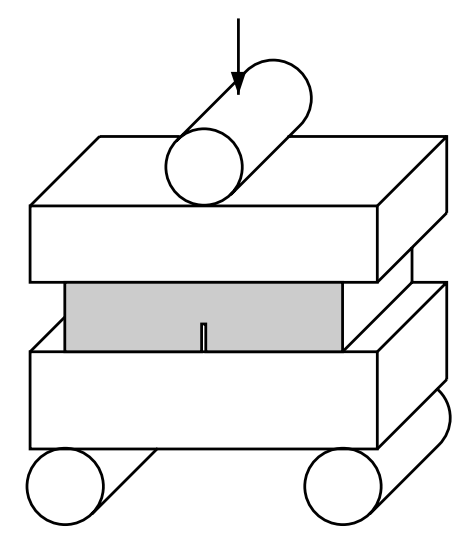

Figure 1. Schematic view of the SB bend test. The observation face of the SiC sample is shown in gray.

Roux et al. 

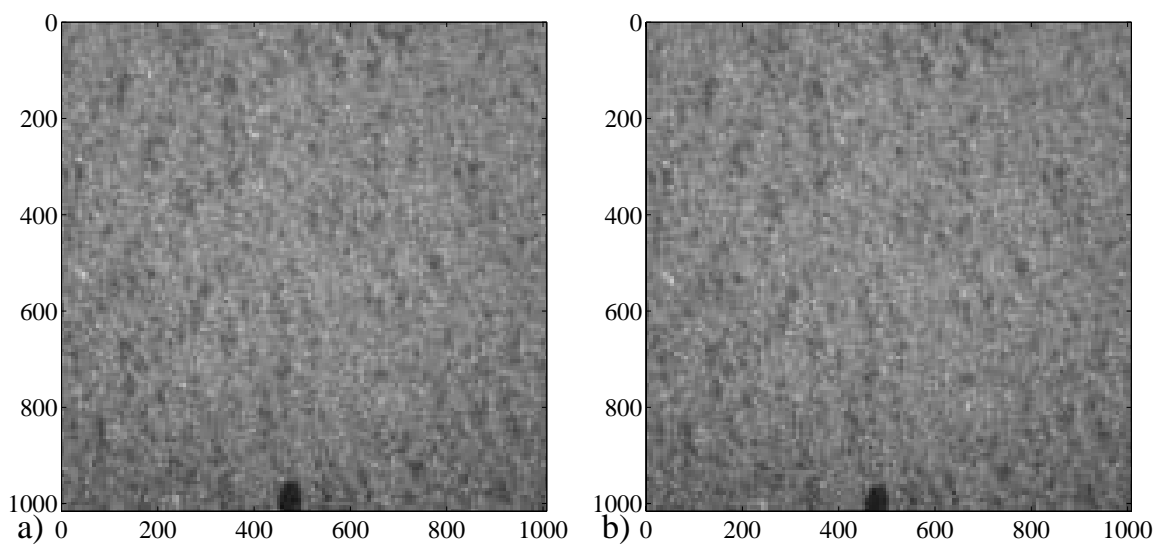

Figure 2. Eight-bit pictures of silicon carbide sample surface before (a) and after (b) loading. The notch on the lower edge of the sample is clearly visible (1 pixel $\leftrightarrow 1.85 \mu \mathrm{m})$.

Roux et al. 

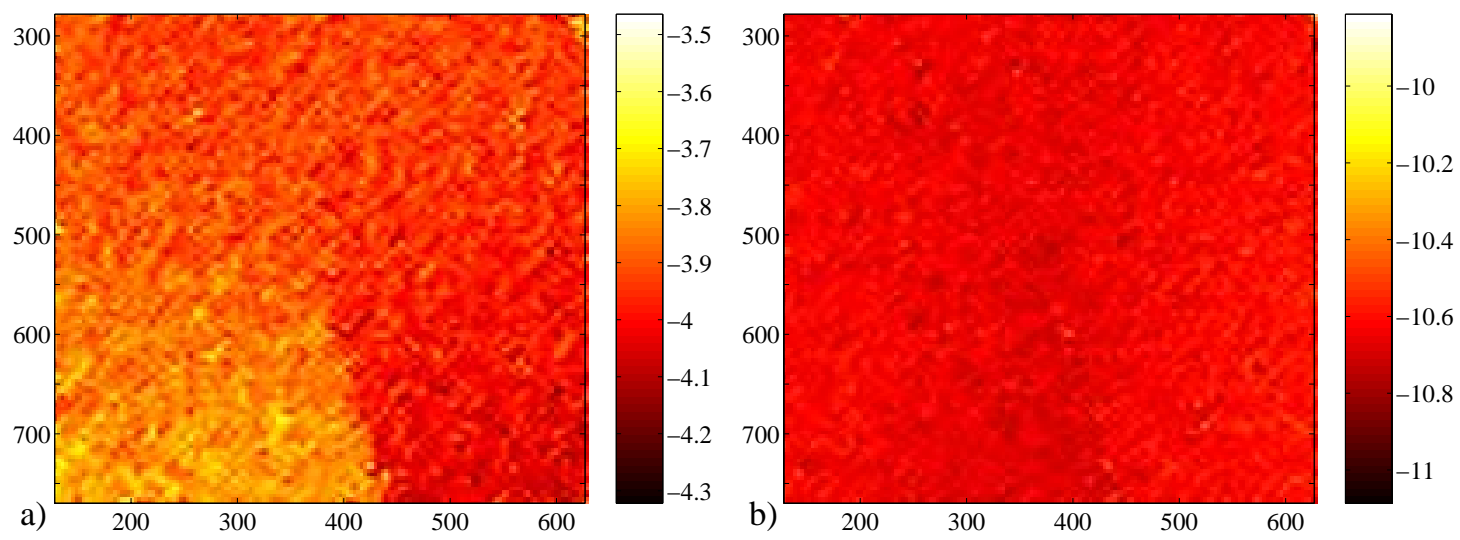

Figure 3. Horizontal (a) and vertical (b) components of the displacement field expressed in pixels and resolved using Q1 elements of size $8 \times 8$ pixels ( 1 pixel $\leftrightarrow 1.85 \mu \mathrm{m})$.

Roux et al. 

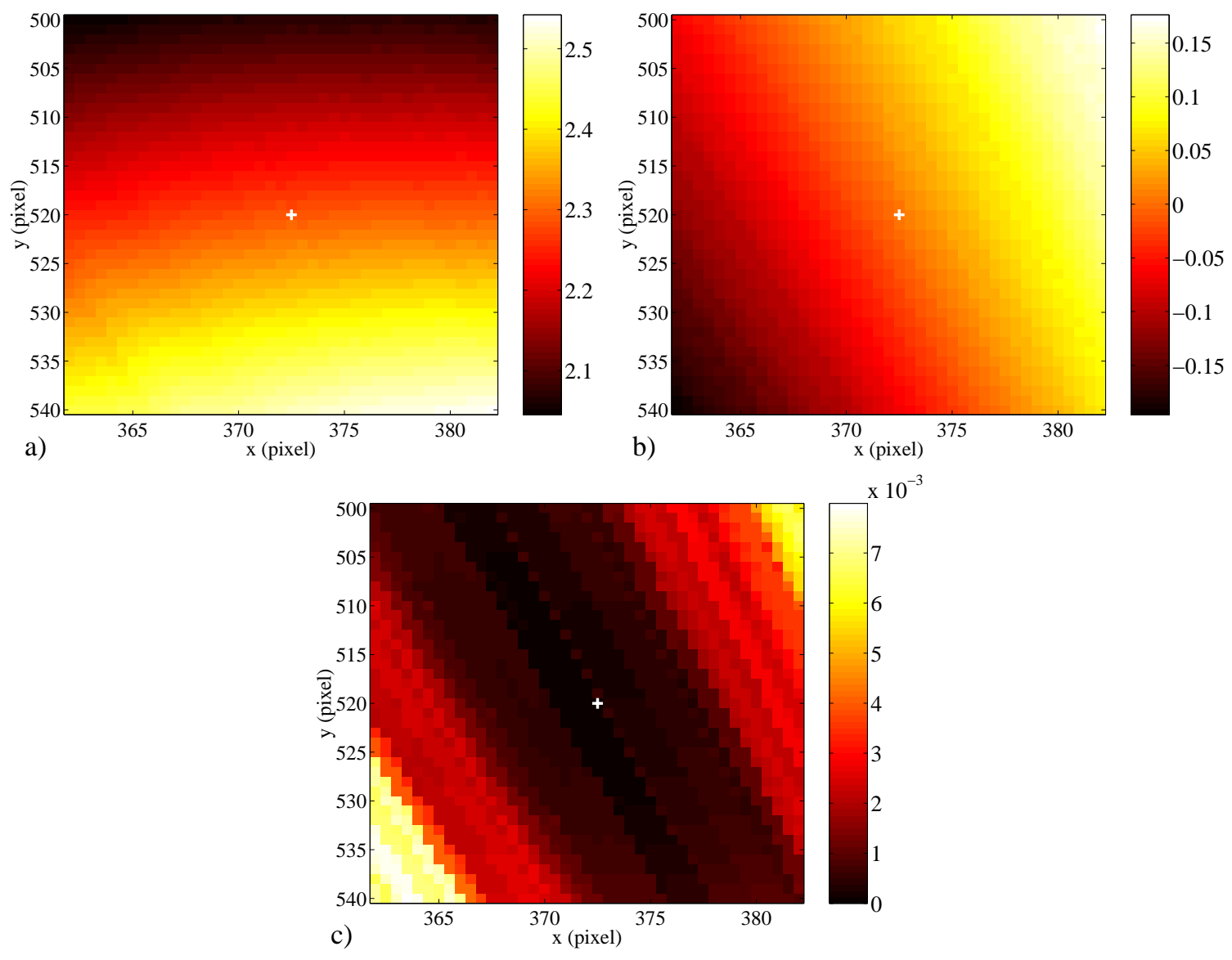

Figure 4. $K_{I}$ in $\mathrm{MPa} \sqrt{\mathrm{m}}$ (a), $K_{I I}$ in $\mathrm{MPa} \sqrt{\mathrm{m}}$ (b) and global residual $\left(\mathcal{T}-\mathcal{T}_{\min }\right) / \mathcal{T}_{\min }(\mathrm{c})$ as functions of the assumed crack tip position $x$ and $y$. The optimal position is depicted by the white cross.

Roux et al. 

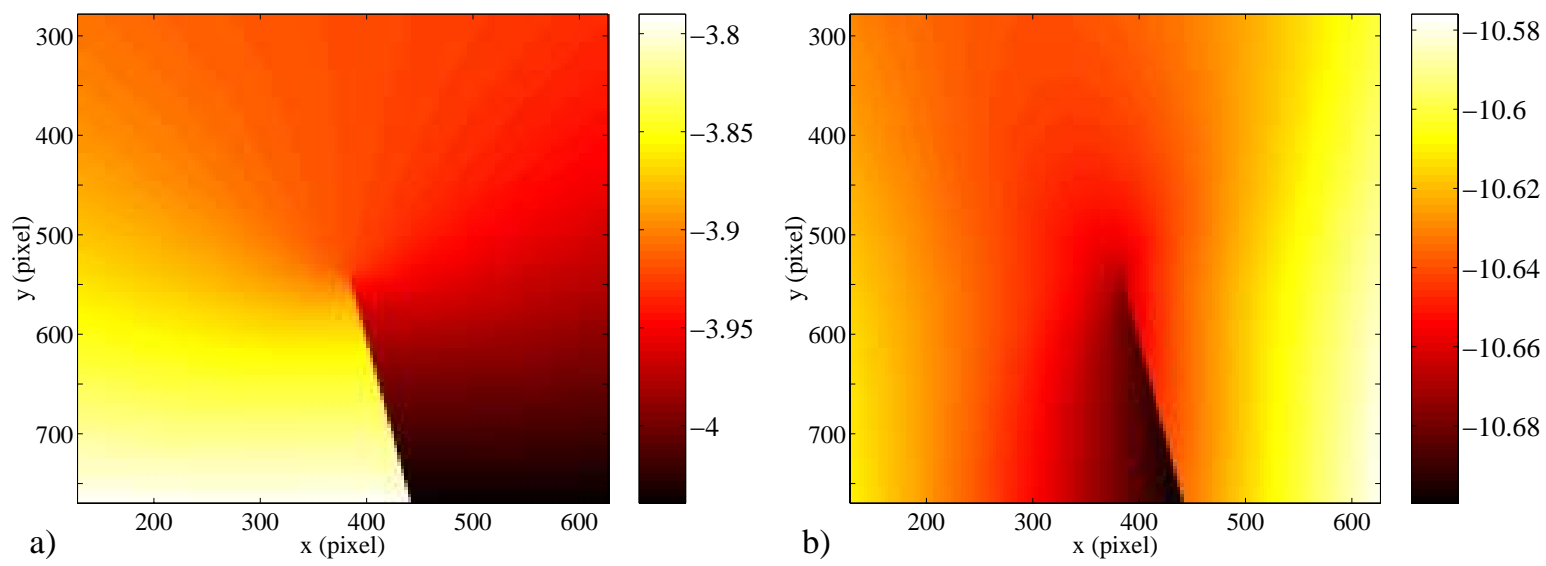

Figure 5. Horizontal (a) and vertical (b) components of the displacement field expressed in pixels as projected onto the basis of suited functions in a post-processing of the measured displacements ( 1 pixel $\leftrightarrow 1.85 \mu \mathrm{m})$.

Roux et al. 

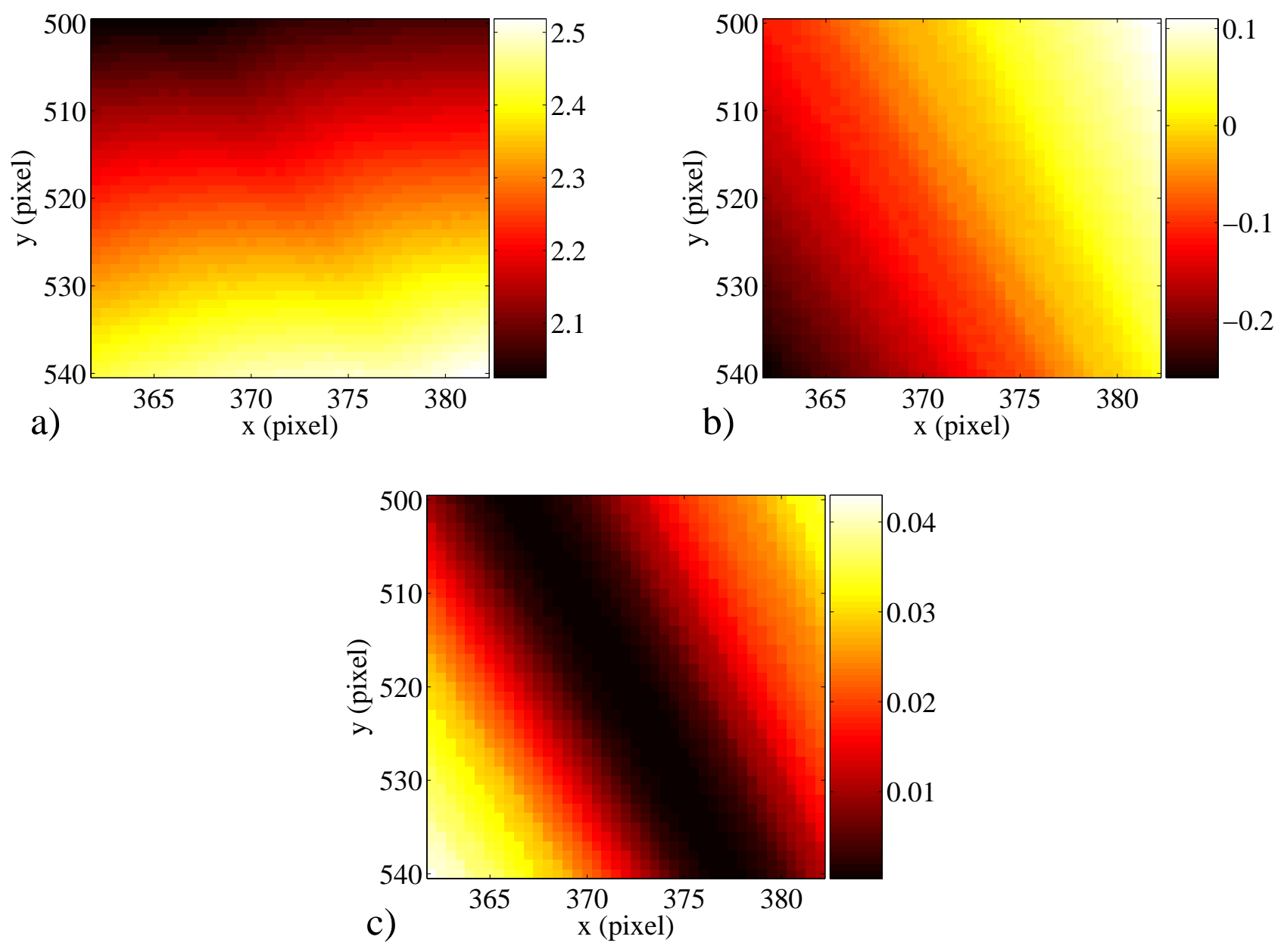

Figure 6. $K_{I}$ in $\mathrm{MPa} \sqrt{\mathrm{m}}(\mathrm{a}), K_{I I} \mathrm{MPa} \sqrt{\mathrm{m}}(\mathrm{b})$ and global residual $\left(\mathcal{T}-\mathcal{T}_{\text {min }}\right) / \mathcal{T}_{\text {min }}$ (c) as functions of the assumed crack tip position $x$ and $y$.

Roux et al. 

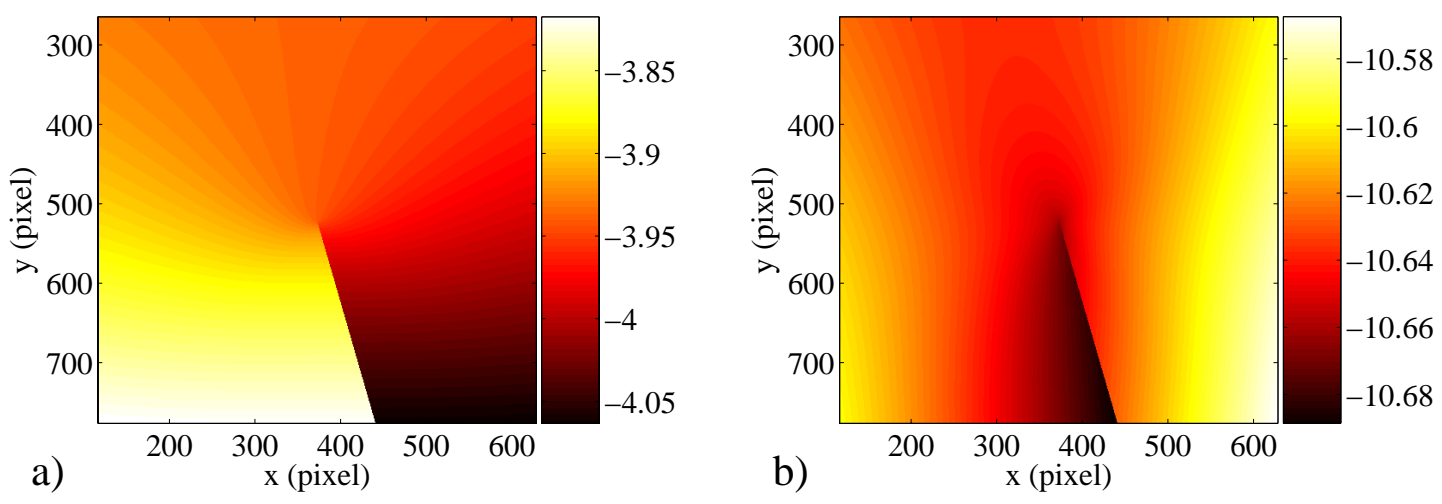

Figure \%. Horizontal (a) and vertical (b) components of the displacement field expressed in pixels and identified by using the integrated approach (1 pixel $\leftrightarrow 1.85 \mu \mathrm{m})$.

Roux et al. 


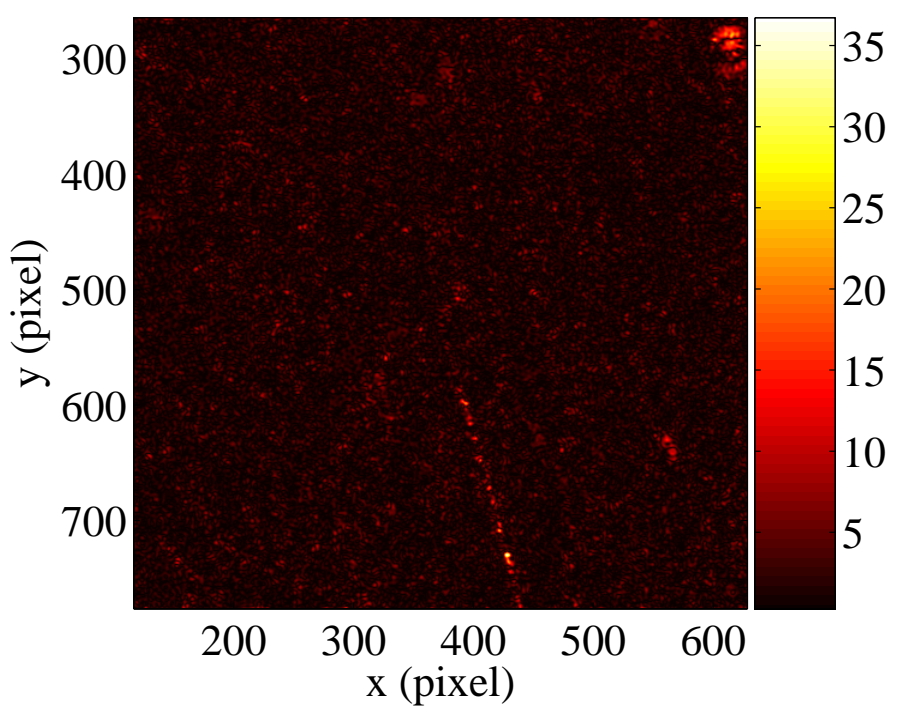

Figure 8. Residual error map for the integrated approach. The spatial integral of the square of this field gives the objective function to be minimized. The mean value of local error is about 1.5 gray levels.

Roux et al.

\section{Address for Offprints:}

Stéphane Roux (corresponding author)

UMR CNRS/Saint-Gobain 125

39 quai Lucien Lefranc, F-93303 Aubervilliers Cedex, France. 